Seit einigen Jahren habe ich mit einer Reihe von Mit. arbeitern versucht, Erfahrungen über die physiologischen Wirkungen des Schilddrüsensekretes zu sammeln, und ich möchte nachfolgend einen kurzen Ueberblick über die bisher vorliegenden Ergebnisse unserer Versuche geben.

Durch die Pathologie angeregt, hat man immer wieder versucht, eine unmittelbare Wirkung der Schilddrüse auf den Kreislauf zu konstatieren. Sehr zahlreiche Versuche, an deren Ausarbeitung die Herren Dr. Flack, Dr. Walther-v. Rodt und Dr. Ossokin beteiligt waren, haben gezeigt, daB das Schilddrüsensekret weder auf den Blutdruck, noch auf die Pulszahl irgendwelchen Einfluß hat. Gleichgiiltig, ob man viel oder wenig Schilddrüsensekret anwendet, bleibt in reinen Versuchen bei intravenöser Injektion, vorausgesetzt, daß diese mit den notwendigen Vorsichtsmaßregeln erfolgt, der Blutdruck auf stets gleicher Höhe, und die Pulszahl wird weder kleiner noch größer.

Bis jetzt wurde vom Schilddrüsensekret gesprochen. In der Mehrzahl der Versuche handelt es sich aber nicht um die Anwendung des noch unbekannten Schilddrüsensekretes, sondern um entweder selbstangefertigten Extrakt aus frischen Schilddrüsen, oder um Extrakte von Schilddrüsentabletten erprobter Herkunft, oder um das Präparat Thyreoglandol der Firma Hoffmann-La Roche \& Co. Das Gleiche gilt von Oswalds vorzüglich wirkendem - da, wo die später zu besprechenden Wirkungen vorhanden sind - Jodthyreoglobulin. Nur da, wo, wie in den Versuchen von Flack, von v. Rodt und mir, die Schilddrüsennerven gereizt wurden, konnte von physiologischem Schilddrüsensekret im wahren Sinne des Wortes gesprochen werden.

Nachdem am Gesamttier diese rein negativen Ergebnisse erhalten worden waren, erwuchs die Aufgabe, die Angelegenheit am isolierten Säugetierherzen zu prüfen. Herr Dr. Richardson sowie Herr Dr. Kakehi konnten am überlebenden Säugetierherzen, welches am Langendorffschen Apparate arbeitete, weder eine Aenderung der Schlagkraft noch der Schlagzahl des Herzens nachweisen. Somit bestätigen diese Versuche, was die Beobachtung am Gesamttier gezeigt hatte.

Aus verschiedenen Gründen konnte man nun der Meinung sein, daß möglicherweise die negativen Ergebnisse daher rührten, daß es sich bei den bisher geschilderten Versuchen um die Herzen normaler Tiere handelte. Aus diesem Grunde schritten wir zur Prüfung des Verhaltens von überlebenden Herzen, die aus Tieren stammten, denen eine gewisse Zeit vor der Anstellung der Versuche die Schilddrüse total entfernt worden war, gegenüber Schilddrüsenpräparaten. Unabhängig davon, ob die Schilddrüse kurze oder lange Zeit vor Anstellung der Versuche entfernt worden war, ergaben die Versuche von Dr. Richardson und Dr. Kakehi, daß die Herzen durch das Schilddrüsensekret vollkommen unbeeinflußt blieben.

Es ist klar, daß diese Ergebnisse eine Bedeutung für die

Aus dem Physiologischen Institut der Universität in Bern.

\section{Die physiologischen Wirkungen des Schilddrüsensekretes und Methoden zu ihrem Nachweis.}

\section{Von Leon Asher.}

Es kann nicht genug betont werden, daß unsere Kenntnisse über die tatsächlichen physiologischen Wirkungen des Schilddrüsensekretes nicht sehr groß sind und der nötigen Bestimmtheit ermangeln, welche wir beispielsweise hinsichtlich der Wirkungen des Adrenalins besitzen. Die reichen Erfahrungen der Pathologie, die Erfolge der klinischen Behandlung, die scheinbare Uebereinstimmung zwischen den Theorien über die Schilddrüse und den Geschehnissen im Verlaufe und nach Behandlung vonSchilddrüsenkrankheiten täuschen gar zu leicht den Besitz von Kenntnissen vor, die in Wirklichkeit noch nicht vorhanden sind.

Von Experimentalforschern ist diese Sachlage schon mehrfach hervorgehoben worden; ja, man findet gelegentlich die Ansicht, daß die einzige gesicherte physiologische Wirkung des Schilddrüsensekretes diejenige sei, den Stoffwechsel zu steigern und beim Tier ohne Schilddrüse den herabgesetzten Stoffwechsel wieder zur Norm zurückzubringen. Für jemanden, der sehr kritisch ist, ließen sich sogar gegen diese recht bescheidene Formulierung unserer Kenntnisse Einwände erheben. Es ist klar, daß jeder weitere Fortschritt sowohl der Physiologie als auch der Pathologie an den Erwerb experimentell gesicherter Kenntnisse über die physiologischen Wirkungen des Schilddrüsensekretes geknüpft ist. Möglichkeiten des pathologischen Geschehens besitzen, denn es muß aus ihnen gefolgert werden, daß die bloße Vermehrung des Schilddrüsensekretes an und für sich und das bloße Fehlen des Sekretes die Schlagfähigkeit und die Schlagzahl des Herzens. schwerlich zu ändern vermögen und deshalb Veränderungen, die man bei einer Mehrung oder Minderung des Schilddrüsensekretes beobachtet oder zu beobachten glaubt, auf andere Momente zurückgeführt werden müssen.

Gegenüber den bisherigen negativen Ergebnissen stehen aber ganz bestimmte, experimentell nachweisbare Wirkungen des Schilddrüsensekretes. In Kürze lassen sich diese, ganz allgemein gesprochen, dahin formulieren, daß die Anspruchsfähigkeit des gesamten autonomen Nervensystems, sowohldas parasympathische wie das symathische, durch Schilddrüsensekret abgeändert, richtiger gesagt, gesteigert wird, und zwar handelt es sich dabei um mittelbare Wirkungen, d. h. um solche Wirkungen, die darin bestehen, daß im Gang befindliche Vorgänge in der Art und Weise, wie sie ablaufen, beeinflußt werden, nicht aber die Vorgänge selbst zur Auslösung gebracht werden. Aus diesem Sachiverhalte heraus ist es klar, daß der Weg zur Erkenntnis erst dadurch eröffnet wurde, daß man passende Reaktionen auffand, in deren Ablauf das Schilddrüsensekret mitbestimmend eingreift. Als solche passende Reaktionen erwiesen sich die folgenden: 1. die Erhöhung der Erregbarkeit des N. 
splanchnicus auf elektrische Reizung, 2. die Erhöhung der Erregbarkeit des N. vagus, 3. die Erhöhung der Erregbarkeit des N. depressor. Eine ihrer Intensität und ihrer zeitlichen Dauer nach genau abgestufte elektrische Reizung dieser Nerven hat einen größeren Erfolg, wenn gleichzeitig oder ganz kurz vorher intravenös Schilddrüsenextrakte injiziert werden, oder wenn gleichzeitig die Nerven der Schilddriise so gereizt werden, daß die Schilddrüse vermehrt ihr Sekret an den Kreislauf abgibt. Hier sind also die durch die Nervenreizung geweckten Vorgänge diejenigen, in welche das Schilddrüsensekret modifizierend eingreift. Von noch größerer-Bedeutung und noch größerer Allgemeinheit ist eine andere Reaktion, nämlich die Verstärkung der Wirksamkeit.von Adrenalin. Hat man eine Adrenalindosis von ganz bestimmter Stärke während einer bestimmten, genau abgemessenen Zeit intravenös injiziert, so erhält man eine Blutdrucksteigerung von einer Höhe und einer zeitlichen Dauer, die sich bei richtiger Arbeitsweise unter denselben Bedingungen ziemlich genau reproduzieren lassen. Sobald aber die genau gleiche Dosis von Adrenalin in genau der gleichen Zeit injiziert wird, während gleichzeitig oder kurze Zeit vorher Schilddrüsenextrakte injiziert oder die Schilddrüsennerven gereizt werden, wird eine größere Druckhöhe erreicht und die Dauer des Druckanstiegs verlängert. Diese Reaktion gestattet, den Angriffsort der Wirkung des Schilddrüsensekretes genauer zu präzisieren. Denn nach allem, was wir zurzeit über den Angriffsort des Adrenalins wissen, liegt dieser weder in den Nervenendigungen noch im kontraktilen Protoplasma des Muskels selbst, sondern in einer Zwischensubstanz, für welche ich den Namen neuroplasmatische Zwischensubstanz (receptiv substance nach Langley, myoneural junction nach Elliott) vorgeschlagen habe.

Diese Reaktion, der, wie mir scheint, große Bedeutung zuzumessen ist, kann auch noch auf eine andere Weise nachgewiesen werden, nämlich mit Hilfe der Laewen-Trendelenburgschen Methode.

Durchströmt man die hintere Extremität des Frosches mit einer Ringerlösung und macht man zu dieser einen der Stärke und der Zeit nach genau dosierten Zusatz von Adrenalin, so erhält man als Ausdruck der Ge äßverengerung eine Verminderung der aus der Vene ausfließenden Tropfenzahl. Injiziert man aber gleichzeitig mit den sonst gleichen Bedingungen Schilddrüsenextrakte, beziehentlich Thyreoglandol, so wird die Tropfenzahl viel mehr vermindert, demnach hat das Adrenalin eine stärkere Verengerung der Gefäße herbeigeführt.

Die bis jetzt geschilderten Reaktionen sind zum Teil durch Oswald gleichfalls beobachtet worden. Dieser bediente sich des von ihm entdeckten Jodthyreoglobulins. Er findet, daß die Sensibilisierung der verschiedenen Nervenwirkungen mit seinem Jodthyreoglobulin noch ausgeprägter ist, als wir bei der Verwendung von anderen Schilddrüsenextrakten und bei Reizung der Schilddrüsennerven beobachteten. Er zieht daraus, wie auch aus hier nicht diskutierten klinischen Erfahrungen, den Schluß, daß Jodthyreoglobulin selbst die wirksame Substanz der Schilddrüse sei. Gleich wie wir, hat er sich nicht überzeugen können, daß dem Jodothyrin von Baumann eine markante Wirkung zukomme. Ich halte den Schluß von Oswald nach den neueren Erfahrungen, die wir gemacht haben, nicht für zwingend. Es scheint mir, daß man aus den Beobachtungen von Oswald zunächst mit Sicherheit nur den Schluß ziehen kann, daß er mit seinem Jodthyreoglobulin mehr von der wirksamen Substanz injiziert, als wenn man die anderen Schilddrüsenpräparate benutzt, oder wenn man die Schilddrüsennerven reizt und so die Absonderung kleiner Mengen von physiologischem Sekret hervorruft. Einen weitergehenden Schluß möchte ich vor allem deshalb nicht ziehen, weil das Präparat Thyreoglandol der Firma Hoffmann-La Roche alle bis jetzt geschilderten Reaktionen des Schilddrüsensekretes geliefert hat und dieses Präparat eiweißfrei ist und nur Spuren von Jod enthält. Die Bedeutung dieser Tatsache ist klar, und es wird darauf noch zurückzukommen sein.

Auf Grund der von uns gemachten Erfahrungen schien die Möglichkeit gegeben, ein viel diskutiertes Problem in Angriff zu nehmen, nämlich die Erzeugung von Symptomen vermehrter Absonderung von Schilddrüsen. sekret. Mit Absicht vermeide ich den Ausdruck. Erzeugung von experimentellem Basedow, weil uns die Experimente am Tier nicht gestatten, von Basedowscher Krankheit zu sprechen. Schon die sehr eingeschränkte Aufgabe der
Erzeugung von Hypersekretion begegnet großen Schwierigkeiten, weil bekanntlich es bisher nicht gelungen ist, im Blute von Tieren oder Menschen das Sekret der Schilddrüse nachzuweisen, d. h. es ist bisher keinem Experimentator gelungen, im Blute aus der Schilddrüse Jodothyrin oder Jodthyreoglobulin festzustellen. Natürlich, wenn keine der beiden Stoffe, wie es anfängt wahrscheinlich zu werden, das Schilddrüsensekret ist, $\mathrm{mu} ß$ der Versuch zur Aussichtslosigkeit verurteilt sein. Durch die Versuche von Flack und mir, sowie diejenigen von v. Rodt und von Ossokin halte ich zwar den biologischen Nachweis von Schilddrüsensekret im Blute und der physiologischen Bedingungen für erbracht, aber weitere Nachweise wären sehr erwünscht, vor allem solche, welche technisch mit weniger großen Schwierigkeiten verknüpft sind, als die hier erwähnten Versuche.

Auf folgende Weise ist durch 'die mit" größter Sorgfalt und Aufwand von viel Mühe von Herrn Privatdozent Dr. Eiger. ausgefïhrten Versuche, über welche ich schon vorläufig in der Sitzung des Medizinisch-pharmazeutischen Bezirksvereins Bern vom 3. Juni 1915 (Schweiz. Korr.Bl. 1915 Nr. 37) berichtet habe, der Nachweis der Erzeugung einer vermehrten Menge von Absonderung des Schilddrüsensekretes in das Blut und damit natürlich der Nachweis von Schilddrüsensekret im Blute allgemein gelungen.

Kleine Mengen von Piasma von Rattenblut oder von Rattenblut selbst, welches mit Hirudinzusatz c der mit Oxalatzucatz aufgefangen wurde, haben keinen Einfluß auf die Gefäße des Frusches, wenn diese nach der Methode von Laewen-Trendelenburg durchströmt werden, immer unter der Voraussetzung, daß man nur kleine Mengen, beispielsweise $1 \mathrm{ccm}$ oder weniger, injiziert. Auch die Wirkung von Adrenalin wird durch das gleiche Blut nicht verstärkt. Ganz anders aber verhält es sich, wenn man eine Zeitlang die Ratten mit Schilddrüsentabletten füttert. Die gleiche Menge von Blut, welche von der normalen Ratte keinen Einfluß auf die Wirksamkeit des Adrenalins besitzt, verstärkt, wenn sie von einer mit Schilddrüsentabletten gefütterten Ratte stammt, in sehr merklicher Weise die Wirksamkeit des Adrenalins, die Tropfenzahl, welche aus der Vene ausflie@t, wird wesentlich geringer.

Hiermit ist der Nachweis geliefert worden, daß infolge der Fütterung von. Schilddrüsentabletten soviel mehr Schilddrüsensekret im Blute kreist, daß man es biologisch-experimentell nachweisen kann. Damit dieser Nachweis gelingt, muß die Fütterung der Tiere mit Schilddrüsentabletten in einer von Herrn Dr. Eiger eigens ausgearbeiteten Weise bewerkstelligt werden, worüber er in seiner demnächst erscheinenden ausführlichen Arbeit berichten wird.

Bekanntlich ist früher behauptet worden, daß das Blut von Basedow-Kranken einen vermehrten Adrenalingehalt besäße. Diese Behauptungen wurden als unzutreffend erkannt, nachdem man durch die Arbeit von O'Connor in Gottliebs Institut die Erfahrung gemacht hatte, daß die adrenalinähnliche Wirkung von Blut im Plasma nicht vorhanden ist, sondern erst entsteht, wenn das Blut durch den Gerinnungsvorgang sich verändert hat und eine noch unbekannte Substanz, welche nicht Adrenalin ist, entstanden ist. Die soeben mitgeteilte Entdeckung gestattet, die Angelegenheit von neuen Gesichtspunkten wieder aufzunehmen, denn jetzt kann man an die Möglichkeit denken, daß das Blut von Basedow-Kranken im Gegensatz zum normalen Blut Gefäßverengerung hervorruft, nicht, weil vermehrtes Adrenalin in diesem Blute ist, sondern weil das in ihm befindliche vermehrte Schilddrüsensekret die Wirksamkeit von etwa vorhandenen Spuren von Adrenalin aktiviert. In einem früheren gelegentlichen Versuche haben Dr. v. Rodt und ich im Blute eines Basedow-Patienten diese aktivierende Wirkung, geprüft durch den Blutdruckversuch am Kaninchen, nachweisen können: aber erst die systematischen Versuche von Dr. Eiger mit einer ganzen Anzahl Blutproben verschiedener Basedow-Patienten haben durch Anwendung der hierzu besonders geeigneten Laeven-Trendelenburgschen; Methode gelehrt, daß im Blute dieser Patienten das vermehrte Schilddrüsensekret nachweisbar ist, während im Blute von Normalmenschen und von Menschen mit anderen Schilddrüsenerkrankungen dieser Nachweis nicht gelingt. In seiner ausführlichen Arbeit wird Herr Dr. Eiger den experimentell-biologischen Nachweis der Basedow-Erkrankung eingehender darlegen.

Es dürfte sich empfehlen, wenn'Kliniker die Gelegenheit wahrnehmen wollten, das ihnen zur Verfügung gestellte Material zur Klärung der Sachlage beizuziehen, damit man einen Ueber- 
blick darüber gewinnt, ob der experimentelle Nachweis von vermehrtem Schilddrüsensekret im Blute von Basedow-Patienten eine Bereicherung des diagnostischen Rüstzeuges darstellt. Die Methodik ist nicht so schwer, daB sie nicht in den Laboratorien der Kliniken ausgeführt werden könnte. Das Verfahren der Laewen-Trendelenburgschen Methode ist durch Gottliebs Darstellung in Abderhaldens Handbuch der biochemischen Arbeitsmethoden allgemein zugänglich. Besondere Sorgfalt ist erforderlich bei der Gewinnung des Basedow-Blutes und des normalen Kontrollblutes, damit auch nicht die geringste Spur von Fibrinbildung eintritt.

Außer den bisher beschriebenen Reaktionen auf Schilddrüsensekret habe ich noch über einige neue Reaktionen zu berichten, welche auf den ersten Blick hin etwas anderer Art zu sein scheinen als die bisher geschilderten. Herr cand. med. Streuli konnte in meinem Institute zeigen, daß Schilddrüsenextrakte, wie auch das Thyreoglandol von Hoffmann-La Roche, die überlebende, automatisch sich kontrahierende Blase des Säugetieres in ihren Kontraktionen verstärkt und auch den Tonus der Blase vermehrt. Als wir diese Beobachtung gemacht haben, bezeichneten wir diese Reaktion als den ersten genauer bekannten Fall von unmittelbarer Wirkung des Schilddrüsensekrets. Seitdem sind noch einige ähnliche Erfahrungen gemacht worden. Im Laboratorium von Guggis berg in Bern sind Beobachtungen gemacht worden, welche dafür sprechen, daß das Schilddrüsensekret die automatischen Bewegungen des Uterus zu fördern vermag. In jüngster Zeit hat Herr Dr. Eiger in meinem Institute zeigen können, daß die automatischen Bewegungen des überlebenden, nach dem Verfahren von Magnus arbeitenden Säugetierdarmes durch Schilddrüsenextrakte und durch. Thyreoglandol gefördert werden und daß eine starke Steigerung des Tonus durch diese erzeugt wird. Wenn wir diese Reaktionen als unmittelbare bezeichneten, so geschah es deshalb, weil man nicht experimentell eine andere Reaktion in Gang zu setzen hatte, wie bei den früheren Reaktionen, um an der Aenderung der primären Reaktionen die nur mittelbare Wirkung von : Sehilddrüsensekret zu erkennen. Es könnte daher scheinen, daß man die Auffassung, welche ich früher von der Schilddrüsenwirkung vertreten habe, nämlich die, daß das Schilddrüsensekret ausschließlich eine mittelbare Wirkung besitze, eine Wirkung, die sich vor allem in der Sensibilisierung der neuroplasmatischen Zwischensubstanz äußere, zu verlassen habe, aber bei näherem Zusehen ist das nicht der Fall. Denn die soeben geschilderten und jederzeit demonstrierbaren W:irkungen lassen sich nur dann nachweisen, wenn automatische Organe sich in einem Zustande der Tätigkeit befinden, um so besser, je günstiger dieser Zustand ist. Beispielsweise ist man nicht imstande, die geringste Wirkung an der überlebenden Säugetierblase zu erzielen, wenn diese sich im Zustande der Ruhe befindet. Nur wenn sie den zur Automatie günstigen $\mathrm{Zu}-$ stand des mittleren Tonus und Automatie besitzt, kann man mit Sicherheit die Wirkung der Schilddrüse nachweisen. Daraus ergibt sich, daß der ganze Unterschied gegenüber den früheren Reaktionen darin besteht, daß wir das Schilddrüsensekret auf einen natürlichen Erregungsvorgang in den automatischen Organen einwirken lassen, der in seiner Ablaufsart durch das Schilddrüsensekret modifiziert wird. Deshalb glaube ich, daß. man die Lehre noch zu Recht bestehen lassen kann, daß die allgemeinste Wirkung des Schilddrüsensekretes in einer Sensibilisierung oder Aktivierung oder, wenn man nichts präjudizieren will, in einer Förderung der: Vorgänge in der neuroplasmatischen Zwischensubstanz der autonomen Organe insgesamt besteht. Vermutlich dürften sich auch die Stoffwechselwirkungen diesem Prinzipe unterordnen, denn die Stoffwechselwirkungen, um welche es sich handelt, sind ja: ausschließlich Vorgänge, die auch ohne Zutun der Schilddrüse im Organismus ablaufen.

Manche von den geschilderten Schilddrüsenwirkungen sind nicht spezifischer Natur, sondern lassen sich auch durch andere: Hormone und pharmakologisehe Stoffe hervorrufen. Solange man das spezifische Schilddrüsensekret nicht in reiner Form kennt, besteht daher eine gewisse Unsicherheit, ob nicht anhaftende Stoffe die Wirkung hervorrufen : und nicht das spezifische Sekret. Dieses Bedenken verliert aber viel an seiner Kraft, seitdem es gelungen ist, durch Reizung der Schilddrüsennerven dieselben Wirkungen zu erzielen, wie durch Schilddrüsenextrakte. Wir glauben, mit Hilfe des interessanten Präparates der Firma Hoffmann-La Roche dem Wesen der wirksamen Sub$\operatorname{stanz}$, die, wie andere Hormone, kein Eiweißkörper zu sein scheint, auch kein Ferment und kein Jodkörper, näherzukommen. Erst wenn die in meinem Laboratorium in Gang befindliche Untersuchung von Herrn Privatdozent Dr. Abelin über die Stoffwechselwirkungen des Thyreoglandols zum Abschluß gebracht. worden sind, werde ich Näheres hierüber berichten. Ich bin aber in der Lage, vorläufig. mitzuteilen, daß Thyreoglandol die gleiche Wirkung wie Schilddrüsentabletten auf den Stoffwechsel besitzt. Diese Feststellung von Herrn Dr. A belin bedeutet eine neue Phase in der Lehre von der Schilddrüse.

Zusammenfassung. 1. Mit Hilfe der Reizung der Schilddrüsennerven wird bewiesen, daß gewisse biologische Reaktionen, die man mit Schilddrüsenpräparaten erhält, auf ihrem Gehalt an dem inneren Sekret der Schilddrüse beruhen.

2. Eine der wichtigsten physiologischen Funktionen des inneren Sekrets der Schilddrüse besteht in Erhöhung der Anspruchsfähigkeit des sympathischen und parasympathischen Nervensystems. Der Angriffsort der Sensibilisierung liegt in der neuroplasmatischen Zwischensubstanz, welche die Verbindung zwischen autonomen Nerven und dem Protoplasma herstellt.

3. Die automatischen Bewegungen des überlebenden Darmes und der überlebenden Blase von Säugetieren werden durch Schilddrüsensekret verstärkt.

4. Für praktische Zwecke ist die brauchbarste Reaktion zum Nachweis von Schilddrüsensekret die Verstärkung der Adrenalinwirkung am Laewen-Trendelenburgschen Präparat. Mit Hilfe dieser Methode gelingt es, im Blute echter BasedowFälle das Vorhandensein vermehrten Schilddrüsensekrets nachzuweisen. Ferner gelingt es mit Hilfe dieser Methode, den Nachweis zu führen, daß durch Fütterung von Ratten mit Schilddrüsentabletten vermehrte Bildung :von Schilddrüsensekret erzeugt wird.

5. Die biologisch wichtigsten Reaktionen werden nicht allein durch Schilddrüsenextrakte, sondern auch durch das eiweißfreie und fast jodfreie Thyreoglandol erhalten.

6. Das eiweißfreie, fast jodfreie Präparat Thyreoglandol hat die gleiche Stoffwechselwirkung wie vollwertige Schilddrüse. 